\title{
The Influence of Obstructions of Evacuation Node on Subway Stations Fire Evacuation Capability
}

\author{
Ying Huang, Jun Lei, Ke Wang
}

School of civil engineering, Xi' an University of Architecture \&Technology, Xi'an 710055, China

\section{疏散节点障碍物对地铁火灾疏散能力的影响研究}

\author{
黄莺, 雷俊, 王辑 \\ 西安建筑科技大学土木工程学院, 西安 710055 , 中国
}

\begin{abstract}
At present, the subway has become one of the main modes of transportation in China's large and medium-sized cities, and the frequent occurrence and loss of subway fire accidents have attracted much attention in the study of subway fire evacuation. Subway platforms, staircases, escalators, brake machines and so on are the key nodes of fire evacuation. When the evacuation node has obstacles, it will seriously affect the overall fire evacuation ability. Through the pathfinder evacuation simulation software for typical subway station fire evacuation node obstacles exist situation simulation, it is concluded that the evacuation of node flow rate and the evacuation time and so on data, analyze the obstacles exist influence on safe evacuation capacity, thus performance-based fire design for subway station optimization, and provide reasonable suggestions for the establishment of the fire safety evacuation plan.
\end{abstract}

Keywords: pathfinder; subway fireevacuation;obstructions; evacuation node

\section{摘要}

目前, 地铁已成为我国大中城市主要交通 方式之一, 地铁火灾事故频发且损失巨大, 使 得地铁火灾疏散研究备受关注。地铁中站台、
楼梯、自动扶梯、闸机、出入口通道等是火灾 疏散必经的关键节点, 当疏散节点存在障碍物 时会对严重影响整体火灾疏散能力。本文通过 pathfinder 疏散模拟软件对某典型地铁站火 灾发生时疏散节点存在障碍物进行情形模拟, 得出各疏散节点的流出速率和疏散时间等数 据, 分析障碍物存在对安全疏散能力的影响, 进而为地铁站性能化火灾设计的优化和火灾 安全疏散的制定提供合理建议。

关键词: pathfinder; 地铁火灾疏散; 障碍物; 疏散节点

\section{1. 引言}

随着轨道交通技术的快速发展和日渐成 熟, 地铁快捷、平稳特点的已使其成为大城市 人口出行的重要的交通工具, 在其带来便捷的 同时, 地铁的安全性能也备受关注。地铁火灾 作为主要地铁安全事故之一, 其发生时的安全 疏散能力也成为地铁安全性能的研究重点。地 铁火灾产生原因错综复杂、发生频率高、造成 人员伤亡大、经济财产损失大，据统计 2004 年 2017 年中国国内共发生有伤亡事故的地 铁火灾共 8 次，其中 2017 年在香港 8 号线由 于人为纵火造成的地铁火灾导致 17 人受伤其 中 2 人病危、 2009 年上海 11 号线由于电气原 因产生的地铁火灾导致 1 人死亡 6 人受伤 ${ }^{[1]}$ 。 由于地铁结构复杂、空间密闭、人员密度大的 特点, 致使地铁火灾疏散难度大、灭火救援难 度大、造成伤亡 ${ }^{[2]}$ 。地铁火灾疏散具有自下 
而上的特点, 且地铁列车内人流量大, 所以导 致地铁站下层人数占大部分, 地铁火灾发生时 的关键疏散节点依次为站台、楼梯、自动扶梯、 闸机、出入口通道。在地铁火灾发生时, 在疏 散节点上跌倒的行人、丢弃的行李、不合理的 建筑设计和设施摆放等形成障碍物造成道路 拥堵或堵塞, 削减了地铁整体安全疏散能力。 连续模型的 (Agent-based) pathfinder 充分 考虑了人员与障碍物的碰撞, 能更好的模拟真 实紧急疏散情况, 在国际上疏散运用中较为普 遍 ${ }^{[3]}$ 。论文以某典型地铁站为例, 通过应用 pathfinder 疏散模拟软件的 steering 模式 ${ }^{[4]}$, 对火灾发生时疏散节点存在障碍物的疏散进 行模拟, 对人员流出速率和疏散时间等关键数 据进行分析对地铁火灾紧急疏散管理提供合 理的建议。

\section{2. 地铁火灾疏散特点}

地铁火灾发生时, 由于地铁只能自下而上 疏散, 人员疏散路径一般依次为列车、站台、 楼梯或自动扶梯、站厅、闸机、出入口通道。 由何理 ${ }^{[5]}$ 在地铁车站大客流疏散分析中表明 属于疏散关键点处的站台, 楼梯, 自动扶梯, 闸机, 入口处通道, 出入口处楼梯和自动扶梯 所处空间狭小, 加之地铁疏散节点本身具有的 动态性、不稳定性和突发性等特点, 导致在地 铁疏散节点极易发生拥堵。由 D. Helbing ${ }^{[6]}$ 等人提出的社会力模型描述出当行人的期望 速度增加到某一值时, 行人之间的相互阻力将 会增大, 成功解释了群体效应与快即是慢效应, 此时堵塞越来越严重, 而这种现象发生在关键 疏散节点会严重地削弱地铁火灾疏散能力。 Bryan J. L. ${ }^{[7]}$ 对国外地铁火灾中成功疏散的人 员的疏散路径和紧急情况的反应进行了调查 分析, 为地铁疏散仿真模型建立的提供了理论 数据的支撑。由何理 ${ }^{[8]}$ 等人对国内地铁乘客的 在突发事件下行为与心理特征进行调查和分 析, 以及 Jinxing Ying ${ }^{[9]}$ 等人用动态规划法对 不同人群在疏散过程中的流动性进行了分析, 为地铁疏散提供了现实理论数据指导。

\section{1 地铁站台疏散特点}

站台作为车厢内人员从车厢内向外逃离 的第一个通道, 它具有良好的水平疏散能力, 能较好的疏散人群, 但同时它的疏散能力受地
铁站台处屏蔽门的影响。当地铁火灾发生时, 人员首先从车厢内撤离, 由于车厢内乘客众多 而车厢门狭窄, 在站台的车厢门处形成第一个 拥堵节点, 特别是在乘客乘车高峰期。疏散人 员开始寻找逃离路径时, 与地铁车门处站台上 的人员极易形成人员交汇，造成拥挤，同时站 台与车厢之间的间隙更易在拥堵时造成人员 踏空或绊倒。

\section{2 地铁自动扶梯疏散特点}

自动扶梯由梯路和两旁的扶手构成, 自动 扶梯的倾角按照《地铁设计规范》规定设为 $30^{\circ}$, 其运行速度宜采用 $0.65 \mathrm{~m} / \mathrm{s}$, 自动扶梯 作为持有行李和行动不便的老弱病残孕乘客 首选的乘坐工具, 由于自动扶梯的梯面比较狭 窄, 只能允许 1 2 人并排通行, 当乘客带有 大件行李时仅可允许一人通行 ${ }^{[10]}$ 。

地铁火灾发生时, 自动扶梯关闭, 此时上 行和下行自动扶梯均可作为疏散通道以提高 紧急疏散的效率。自动扶梯作为垂直疏散通道 [11], 人群在其上行走或奔跑的速率比水平通道 慢且更耗费体力, 容易在惊慌的境况下抛弃行 李或跌倒。自动扶梯的下端由于下层人员的聚 集造成拥堵减小自动扶梯的疏散速率, 拥堵的 人群也易造成跌倒和踩踏事故。

\section{3 地铁楼梯疏散特点}

地铁中的楼梯一般分为有休息平台楼梯 和无休息平台的楼梯, 按形状也可分为直行和 $\mathrm{T}$ 型。地铁车站中楼梯常与自动扶梯作为垂直 疏散通道配合使用, 其疏散特点也类似于自动 扶梯, 楼梯每踏步的宽度一般大于自动扶梯, 整体的疏散能力比自动扶梯好, 允许 2 3 人 并排通行 ${ }^{[12]}$ 。当发生火灾事故时, 下层众多的 人员都会汇聚于楼梯下端, 由于恐慌造成人员 的推挤都极易消耗疏散人员的体力, 使部分人 产生脱力和摔倒现象, 形成障碍物阻塞楼梯段 下端, 降低疏散效率, 甚至是导致踩踏事故。

\section{4 地铁闸机疏散特点}

我国地铁车站大多数使用门扉式闸机，闸 机作为乘客唯一从站内向出入口疏散的通道, 当火灾发生时闸机开启形成狭小的单人通道 也减缓了疏散效率造成后方的拥堵 ${ }^{[13]}$ 。闸机处 
的拥堵会进一步的减小闸机的疏散效率, 不论 是疏散人员摔倒或者其他障碍物的堵塞, 都大 大降低闸门的疏散效率, 容易造成更为严重的 踩踏事故。

\section{3. 地铁疏散节点受阻的风险}

疏散节点的障碍物主要源于跌倒的行人, 丢弃的行李, 以及不合理的建筑设计和设施摆 放。这些障碍物的产生都会增加疏散节点受阻 的风险。

(1) 跌倒的行人形成的障碍物。在客流 量高峰时段, 拥堵现象明显, 尤其是在疏散节 点上人员滞留排队现象明显。由于地铁乘客人 流量大、人员构成复杂, 其中不乏老弱病残孕 的乘客。火灾发生时, 由于恐慌和不安, 乘客 在奔跑和拥堵的过程中由于体力不支造成跌 倒。此时在保证跌倒人员安全情况下, 人群会 自动避开和绕行。

(2) 丢弃的行李形成障碍物。根据王春 雪 ${ }^{[14]}$ 在北京地铁中乘客人员特征分析中: 在人 流量高峰时段携带行李的乘客为 $73 \%$, 其中携 带大件行李为 9\%; 平峰时段携带行李的乘客 为 $74 \%$, 其中携带大件行李为 $39 \%$ 。无论是客 流量高峰时期还是平峰时期, 携带行李的乘客 数量成为乘客总人数的主要组成部分, 而这些 乘客在地铁火灾发生时, 由于拥堵产生恐慌心 理, 为尽快逃离火灾现场而抛弃自己的行李, 在疏散节点上形成了障碍物。

(3) 不合理的建筑设计和设施摆放。不 合理的建筑设计中通常将尺寸较大的柱或其 他大型建筑物设计在疏散节点的关键位置上, 使得疏散通道更易受阻。设施的不合理摆设也 增加了疏散通道受阻风险, 及如广告牌、花盆、 清洁工具等的设施摆放在疏散节点通道上形 成障碍物, 从而导致人员疏散受阻。

\section{4. 基于疏散节点特点的地铁站模型建立与 分析}

根据上述的疏散节点特点, 建立某典型普 通无换乘地铁站, 设置的疏散关键节点有站台, 4 处自动扶梯, 1 处 T 型楼梯, 4 处闸机通道 组。当火灾发生时, 自动扶梯关闭, 闸机通道 打开, 人员开始疏散。模型只考虑乘客的疏散, 未考虑其余办公室和设备间对疏散的影响。模 拟当乘客遇到火灾时从地铁站厅自动门处开 始疏散, 疏散路径按照站台、楼梯或者自动扶
梯、闸机、出入口通道疏散。

\section{1 典型地铁车站模型的参数设置}

\subsection{1 典型地铁车站的设施参数}

工程概况: 车站总面积 14492.6 平方米, 其中出入口、疏散通道的建筑面积为 20223.78 平方米, 车站总长 $270.6 \mathrm{~m}$, 标准段宽 $19.70 \mathrm{~m}$ 车站设置四个出入口, 车站层数为 2 层。模型 中只考虑乘客行动区域, 其中自动扶梯有限通 行宽度 $116 \mathrm{~cm}, T$ 型楼梯有限通行宽度为 $185 \mathrm{~cm}$, 闸机通道宽 $80 \mathrm{~cm}$, 列车设置 6 道车厢门且宽 为 $260 \mathrm{~cm}$, 出入口通道宽 $610 \mathrm{~cm}$ 。列车载客量 为 1200 人, 地铁站内人数按照 $1.5 \mathrm{~m}^{2} /$ 人设置 共计 2954 人. 疏散人员按 $60 \%$ 肩宽为 $46 \mathrm{~cm}$ 速 度为 $1.3 \mathrm{~m} / \mathrm{s}$ 的男性, 和 $40 \%$ 肩宽为 $41 \mathrm{~cm}$ 速度 为 $1.1 \mathrm{~m} / \mathrm{s}$ 的女性构成 ${ }^{[15]}$ 。

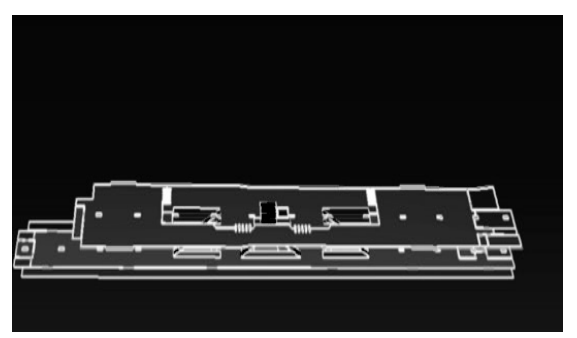

图 1 某地铁车站模型图

\subsection{2 障碍物参数的设置}

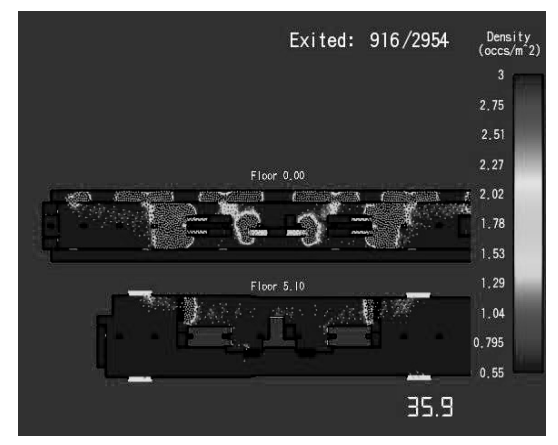

图 $235.9 \mathrm{~s}$ 时的人员密度分布图 


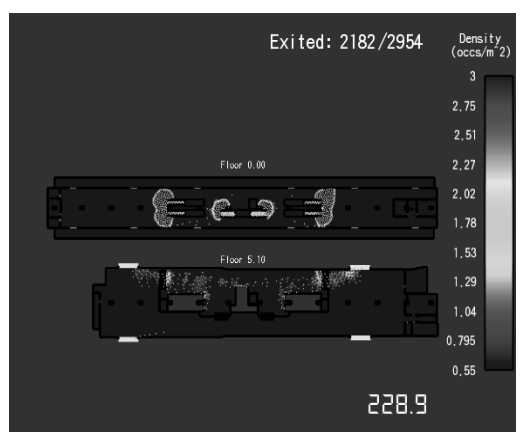

图 $3228.9 \mathrm{~s}$ 时人员密度分布图

根据图 2、图 3, 可得到人员疏散时, 疏 散节点处的人群密度较为集中, 且楼梯和自动 扶梯处的人群最为集中且聚集在疏散节点的 下端。障碍物的位置也会影响该节点的疏散, 当障碍物位于出口中心线时会极大降低疏散 效率 ${ }^{[16]}$ 。故障碍物的设置按照在疏散节点人群 密集处, 障碍物尺寸大小设置为 $50 \mathrm{~cm} \times 50 \mathrm{~cm}$, 分别设置在站台车门处、自动扶梯下端、楼梯 下端、出入通道口处的中心线位置。

\section{2 数据结果分析}

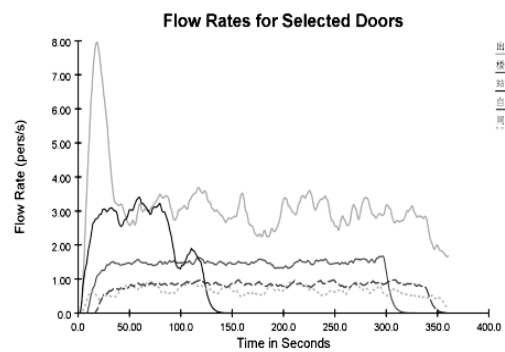

图 4 无障碍物时人员流出速率

由无障碍物时的人员流出速率图得到各 疏散节点人员流出速率和变化情况。人员流出 速率按出入口通道、站台、楼梯、自动扶梯、 闸机依次减小, 在 $150 \mathrm{~s}$ 处站台人员疏散完毕。 出入口通道的流出速率到达一个峰值而后下 降达到平稳, 这是因为在上层人员疏散完毕前 出入口流出速率受上层人员的影响达到一个 峰值, 当上层原有人员疏散基本完毕后出入口 流出速率主要受通过楼梯、自动扶梯向上疏散 的下层人员影响, 而楼梯、自动扶梯向上层疏 散时的流出速率小且稳定, 使出入口通道的流
出速率下降到一个稳定值。

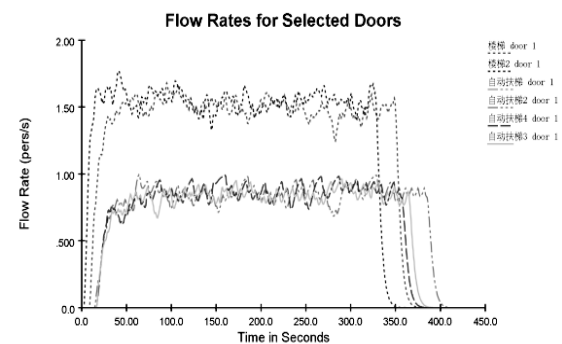

图 5 自动扶梯存在障碍物时人员流出速率

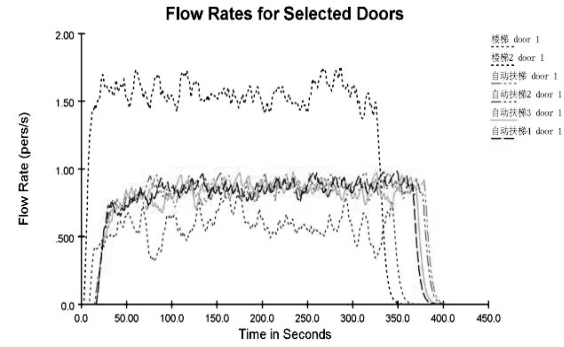

图 6 楼梯存在障碍物时人员流出速率

表 1 无障碍物存在时的疏散结果

\begin{tabular}{|c|c|c|c|}
\hline $\begin{array}{l}\text { 疏散 } \\
\text { 节点 }\end{array}$ & $\begin{array}{l}\text { 通过总人 } \\
\text { 数（人） }\end{array}$ & $\begin{array}{c}\text { 流出速率 } \\
(\text { 人/s })\end{array}$ & $\begin{array}{l}\text { 总疏散时 } \\
\text { 间 (s) }\end{array}$ \\
\hline $\begin{array}{l}\text { 站台 } \\
\text { 车门 }\end{array}$ & 304 & 2. 44 & \\
\hline $\begin{array}{l}\text { 自动 } \\
\text { 扶梯 }\end{array}$ & 264 & 0.84 & \\
\hline 楼梯 & 430 & 1.47 & 356.8 \\
\hline 闸机 & 227 & 0.66 & \\
\hline $\begin{array}{l}\text { 入口处 } \\
\text { 通道 }\end{array}$ & 1182 & 3.32 & \\
\hline
\end{tabular}

表 2 障碍物存在时的疏散结果

\begin{tabular}{ccccc}
\hline $\begin{array}{c}\text { 疏散 } \\
\text { 节点 }\end{array}$ & $\begin{array}{c}\text { 通过总 } \\
\text { 人数 } \\
(\text { 人 })\end{array}$ & $\begin{array}{c}\text { 流出速率 } \\
(\text { 人 } / \mathrm{s})\end{array}$ & $\begin{array}{c}\text { 总疏散 } \\
\text { 时间 }(\mathrm{s}) \text { 间 }(\mathrm{i} \text { 加时 }\end{array}$ \\
\hline $\begin{array}{c}\text { 站台 } \\
\text { 车门 }\end{array}$ & 131 & 0.80 & 358.8 & 2 \\
$\begin{array}{c}\text { 自动 } \\
\text { 扶梯 }\end{array}$ & 0 & 0 & 409.8 & 51 \\
$\begin{array}{c}\text { 楼梯 } \\
\text { 闸机 }\end{array}$ & 568 & 1.51 & 430.9 & 74.1 \\
$\begin{array}{c}\text { 入口处 } \\
\text { 通道 }\end{array}$ & 1330 & 0.00 & 366.3 & 9.5 \\
\hline
\end{tabular}


通过对模拟所得数据整理并进行对比分 析, 可以得到障碍物的存在会使的该疏散节点 人流速率大幅度降低, 同时也会影响到总体的 疏散时间。由于楼梯和自动扶梯作为垂直疏散 节点且宽度小, 布置个数少, 而下层人员又分 布多的情况下, 当某处存在障碍物时就会堵塞 通道, 而其余无障碍物处垂直疏散通道疏散能 力有限, 就会大幅度增加总体的疏散时间。站 台、闸机、出入口通道等水平疏散节点具有宽 度大或布置个数多的优点, 当遇到障碍物时疏 散人群可以选择其他相同节点进行疏散, 此时 对总体疏散时间影响小。

\section{5. 结论}

通过用 pathfinder 对疏散节点处存在障 碍物时的疏散情形进行模拟分析, 可以得出火 灾发生时疏散节点处的障碍物极大的影响着 地铁疏散能力。疏散节点处的障碍物将使该节 点处的人流速率大幅度降低, 并对总体疏散能 力产生影响, 尤其是障碍物处于自动扶梯和楼 梯节点处时会大幅度增加总体疏散时间。鉴于 以上模型模拟和分析, 对地铁火灾安全管理给 出如下建议:

（1）地铁设计者应着重加强对楼梯和自 动扶梯等垂直疏散节点重点优化, 增设垂直疏 散通道或增宽垂直疏散通道的尺寸以增加其 人流疏散效率, 以及加强审图避免影响疏散节 点的建筑设计。

（2）地铁运营管理者应在发生火灾时注 意关注是否有人员的跌倒的情况产生, 并及时 处理以使疏散能够正常的进行。

（3）地铁管理者应加强对站内设施摆 放的合理性进行检查, 及时移走在疏散节点上 影响疏散的设施, 保证疏散路径的畅通。

\section{Acknowledgements}

This study was supported by Construction of shaanxi province science and technology plan projects (2016 - RJ21), xi 'an construction of science and technology plan projects (SJW2017-02), the national natural science foundation of China (51308448)

\section{致谢}

本项目获得了陕西省建设科技计划项目
（2016-RJ21）、西安市建设科技计划项目 (SJW2017 - 02) 、国家自然科学基金 (51308448) 的资助。

\section{参考文献}

[1] 袁勇,邱俊男.地铁火灾的原因与统计分析. 城市轨道交通研究,2014,17(07):26-31+61.

[2] 朱惠军.城市地铁火灾的特点、事故分析 及预防措施.安防科技,2011(03):37-40.

[3] 杜长宝, 朱国庆, 李俊毅.疏散模拟软件 STEPS 与 Pathfinder 对比研究.消防科学 与技术, 2015, 34(04):456-460.

[4] Harold N,Frederick M. Emergency movement,the SFPE hand book of fire protection engineering (3rd edition). Bethesda:Society of Fire Protection Engineers, 2002.

[5] 何理. 地铁车站大客流疏运风险形成机 理及行为特征研究. 北京科技大学, 2016 .

[6] Helbing D,Farkas I,Vicsek T.Simulating dynamical features of escape panic. Nature, 2000, 407: 487-490.

[7] Bryan J L. Human behaviour in fire: The development and maturity of a scholarly study area. Fire and Materials. 1999, 23(6):249-253.

[8] 何理,钟茂华,史聪灵,石杰红. 地铁突发事 件下乘客疏散行为调查研究. 中国安全 生产科学技术, 2009,5(01):53-58.

[9] Ying J X,Li J.Research on emergency evacuation of subway station based on mobile capability. Proceedings of the 2ndInternational Conference on Material Science, Machinery and Energy Engineering,2017,872-875.

[10] 赖丽萍,陈勇烽,晏小波. 国内外地铁车站 楼扶梯设计方法比较分析. 城市轨道交通 研究, 2012,15(10):17-20+42.

[11] 丁元春. 高层建筑人群垂直疏散特性与 疏散策略计算机仿真研究.中国科学技术 大学,2014.

[12] 郭海林,刘宵,王志宁,张利欣.楼梯间障碍 物对安全疏散的影响研究.工业安全与环 保, 2014, 40 (12):28-31.

[13] 田娟荣,周孝清,李健. 地铁自动检票闸机 对人员疏散的影响分析. 火灾科 学,2006(01):38-43+55.

[14] 王春雪,吕淑然,索晓. 基于不同时段人群 特征的地铁疏散仿真研究.中国安全生产 
科学技术, 2015,11(09):101-107.

[15] 徐方,魏东,魏星.公众聚集场所人群疏散 基础数据的分析. 中国安全科学学 报,2008(04):137-145+2.

[16] 王群,王江川,王林.障碍物对人员疏散影 响的分析.工业安全与环保, 2014,40(08):16-17+34. 